\title{
SINGULAR PERTURBATION FOR VOLTERRA EQUATIONS OF CONVOLUTION TYPE
}

\author{
CARLOS LIZAMA AND HUMBERTO PRADO
}

\begin{abstract}
Under the assumption that $A$ is the generator of a twice integrated cosine family and $K$ is a scalar valued kernel, we solve the singular perturbation problem

$$
\left(E_{\epsilon}\right) \quad \epsilon^{2} u_{\epsilon}^{\prime \prime}(t)+u_{\epsilon}^{\prime}(t)=A u_{\epsilon}(t)+\left(K * A u_{\epsilon}\right)(t)+f_{\epsilon}(t), \quad(t \geq 0)(\epsilon>0),
$$

when $\epsilon \rightarrow 0^{+}$, for the integrodifferential equation

$$
w^{\prime}(t)=A w(t)+(K * A w)(t)+f(t), \quad(t \geq 0),
$$

on a Banach space. If the kernel $K$ verifies some regularity conditions, then we show that problem $\left(E_{\epsilon}\right)$ has a unique solution $u_{\epsilon}(t)$ for each small $\epsilon>0$. Moreover $u_{\epsilon}(t)$ converges as $\epsilon \rightarrow 0^{+}$, to the unique solution $u(t)$ of equation $(E)$.
\end{abstract}

\section{INTRODUCTION}

Let $A$ be a closed and densely defined linear operator on a Banach space $X$ and let $K$ be a realvalued function. In this paper we analyze the existence and uniqueness of solutions of the linear Volterra equations of convolution type given by

$$
\left\{\begin{array}{l}
\epsilon^{2} u^{\prime \prime}(t, \epsilon)+u^{\prime}(t, \epsilon)=A u(t, \epsilon)+\int_{0}^{t} K(t-s) A u(s, \epsilon) d s+f(t, \epsilon), \quad t \geq 0 \\
u(0, \epsilon)=u_{0}(\epsilon), \\
u^{\prime}(0, \epsilon)=u_{1}(\epsilon),
\end{array}\right.
$$

and we show that the solution $u_{\epsilon}(t)$ of (1.1) converges as $\epsilon \rightarrow 0$ to the unique solution of

$$
\left\{\begin{array}{l}
w^{\prime}(t)=A w(t)+\int_{0}^{t} K(t-s) A w(s) d s+f(t), \quad t \geq 0 \\
w(0)=w_{0}
\end{array}\right.
$$

The problem concerning the behavior of (1.1) as $\epsilon \rightarrow 0$ is called a singular perturbation problem. Applications frequently occur in linear viscoelasticity theory. For example in the case that $A=\Delta$ then, $\epsilon=\rho$ represents the material density as $\rho \rightarrow 0$; see the monograph by Hrusa and Nohel [11] for more information on the subject. About the singular perturbation problem see $[1,2,4,5,8]$ for recent developments.

In this article we address the question concerning the behavior of (1.1) as $\epsilon \rightarrow 0$ under the assumption that $A$ is the generator of a twice integrated cosine family. In a recent work [8] we have studied this problem under the hypothesis that $A$ is the generator of a cosine family.

To our knowledge the singular perturbation problem when $A$ generates a twice integrated cosine family has not been previously considered in the literature. This case provides many important applications. For instance, an immediate consequence of our main theorem of section 4 occurs when $A=\Delta$

2000 Mathematics Subject Classification. Primary 45N05; Secondary 45D05, 43A15, 47D99.

Key words and phrases. Singular Perturbation, Approximation, $k$-Regularized Families, Integrated Cosine Families.

The second named author is supported by FONDECYT Grant \# 1030007 . 
is the Laplace operator on $L^{p}\left(\mathbb{R}^{N}\right)$. We recall that if $N=1$ and $1 \leq p<\infty$ or $N \geq 2$ and $p=2$ then $A$ is the generator of a strongly continuous cosine family, however in all the other cases this is not longer true. Actually, $A=\Delta$ generates an twice integrated cosine family on $L^{p}\left(\mathbb{R}^{N}\right)$ when $N \leq 5$ and $1<p<\infty$. This result was proved by Keyantuo in [3].

The two main features of this work are, on one side we do not need to assume the existence of solutions beforehand in opposition with the previous works on the subject (see $[4,5]$ ), on the other, we apply the notion of $k$-regularized resolvent families to prove existence and uniqueness of solution when $A$ is the generator of a 2 -times integrated cosine family. Moreover, under the assumption that $A$ generates a twice integrated cosine family we show in section 3 that there is a $k_{\epsilon}$ - regularized resolvent $S_{\epsilon}(t)$ where $k_{\epsilon}:=1 * a_{\epsilon}$ for all $\epsilon \geq 0$, which is twice differentiable and such that the solution of (1.1) is given by the formula

$$
\begin{aligned}
u(t, \epsilon) & =\int_{0}^{t} r_{\epsilon}^{\prime}(t-s) S_{\epsilon}^{\prime \prime}(s) u_{0}(\epsilon) d s+\epsilon^{2} S_{\epsilon}^{\prime \prime}(t) u_{0}(\epsilon)+\epsilon^{2} \int_{0}^{t} r_{0}^{\prime}(t-s) S_{\epsilon}^{\prime \prime}(s) u_{1}(\epsilon) d s \\
& +\int_{0}^{t} r_{0}^{\prime}(t-s)\left(S_{\epsilon}^{\prime \prime} * f_{\epsilon}\right)(s) d s,
\end{aligned}
$$

where $a_{\epsilon}(t)$ and $r_{\epsilon}(t)$ are defined below by equations (3.3) and (4.1) respectively. On the other hand, the key to solve the singular perturbation problem relies on a result due to Lizama [7], about approximation of $k$-regularized resolvent families, which is an extension of the classical Trotter-Kato theorem on convergence and approximation of $C_{0}$-semigroups. The conditions on the kernel $K(t)$ in (1.1) are motivated from some of the known examples which take place in many applications. For instance, if $K(t)=b e^{-a t}$ with $a>0$ and $a+b>0$ then $K(t)$ is shown to satisfy our assumptions. For this and more examples see the monograph [10].

\section{Preliminaries}

We first recall the definition of generator for a $k$-regularized resolvent family; see [6]. Here the symbol ${ }^{-}$denotes the Laplace transform and $\rho(A)$ stands for the resolvent set of the operator $A$.

Definition 2.1. Let $a, k \in L_{\text {loc }}^{1}\left(\mathbb{R}_{+}\right)$be such that there exists $\hat{a}(\lambda)$ and $\hat{k}(\lambda)$ for all $\lambda>\omega$ and $\hat{a}(\lambda) \neq 0$ for $\lambda>\omega$. We will say that a closed linear operator $A$, defined in a Banach space $X$ is the generator of a $k$-regularized resolvent family $\{R(t)\}_{t \geq 0} \subseteq \mathcal{B}(X)$ if

(i) $t \rightarrow R(t) x$ is continuous for all $x \in X$ and there exists $M \geq 0$ such that $\|R(t)\| \leq M e^{\omega t}$, for all $t \geq 0$.

(ii) $\frac{1}{\hat{a}(\lambda)} \in \rho(A)$ for all $\lambda>\omega$.

(iii) $\hat{k}(\lambda)(I-\hat{a}(\lambda) A)^{-1} x=\int_{0}^{\infty} e^{-\lambda s} R(s) x d s$ for all $x \in X$ and all $\lambda>\omega$.

\section{Remark 2.2 .}

It follows from [6, Proposition 3.1], that $R(t)$ commutes with $A$ and satisfies the equation

$$
R(t) x=k(t) x+\int_{0}^{t} a(t-s) A R(s) x d s
$$

for all $x \in D(A), t \geq 0$. Then $R(t)$ is called a $k$-regularized family for the equation

$$
u(t)=f(t)+\int_{0}^{t} a(t-s) A u(s) d s, \quad t \geq 0,
$$

where $f \in C(\mathbb{R} ; X)$. We remark that in the above definition, if $k(t) \equiv 1$ we recover the notion of resolvent family, which is a fundamental concept in the theory of linear Volterra equations, see Prüss 
[10]. In particular, when $a(t)=t$, we obtain the theory of strongly continuous cosine families, related to the abstract Cauchy problem of second order. If $k(t)=t^{2}$ and $a(t)=t$, then $R(t)$ corresponds to a 2-times integrated cosine function.

The following generation Theorem gives the necessary and sufficient conditions on an operator $A$ to be the generator of $k$-regularized resolvent family; see [6] for further details.

Theorem 2.3. Let $A$ be a closed linear unbounded operator with densely defined domain $D(A)$, defined on a Banach space $X$ and let $a \in L_{\text {loc }}^{1}\left(\mathbb{R}_{+}\right)$be satisfying $\int_{0}^{\infty} e^{-\omega t}|a(t)| d t<\infty$. Then $A$ is the generator of a $k$-regularized resolvent family $\{R(t)\}_{t \geq 0}$ such that $\|R(t)\| \leq M e^{\omega t}$ for all $t \geq 0$, if and only if the following two conditions are satisfied

(K1) $\hat{a}(\lambda) \neq 0$ and $\frac{1}{\hat{a}(\lambda)} \in \rho(A)$, for all $\lambda>\omega$.

(K2) $H(\lambda):=\hat{k}(\lambda)(I-\hat{a}(\lambda) A)^{-1}$ satisfy the inequality

$$
\left\|H^{(n)}(\lambda)\right\| \leq M n !(\lambda-\omega)^{-(n+1)}, \lambda>\omega, n \in \mathbb{N}_{0} .
$$

\section{Convergence of Resolvents}

We will consider the problems

$$
u_{\epsilon}(t)=f_{\epsilon}(t)+\int_{0}^{t} a_{\epsilon}(t-s) A u_{\epsilon}(s) d s, \quad t \geq 0, \quad \epsilon \geq 0,
$$

where $f_{\epsilon} \rightarrow f_{0}$ and $a_{\epsilon} \rightarrow a_{0}$ in an appropriate sense as $\epsilon \rightarrow 0$. Assuming the existence of resolvents $R_{\epsilon}(t)$ for (3.1) as well as the stability condition

$$
\sup _{\epsilon>0}\left\|R_{\epsilon}(t)\right\| \leq M e^{\omega t}, \quad t \in \mathbb{R}_{+} .
$$

Then it has been shown in [7] that $R_{\epsilon}(t) \rightarrow R_{0}(t)$ strongly in $X$ as $\epsilon \rightarrow 0$. A particular case of this is the following.

Theorem 3.1. Suppose that $\left\{k_{\epsilon}\right\}_{\epsilon>0} \in L_{\text {loc }}^{1}\left(\mathbb{R}_{+}\right)$and let $\left\{a_{\epsilon}\right\}_{\epsilon>0} \in A C_{\text {loc }}\left(\mathbb{R}_{+}\right)$be of type $(M, \omega), \omega \geq 0$, such that $\hat{a}_{\epsilon}(\mu) \neq 0$ for $\mu>\omega$, and $\int_{0}^{\infty} e^{-\omega s}\left|\dot{a}_{\epsilon}(s)\right| d s<\infty$. Let $A$ be a closed linear and densely defined operator on $X$. Assume that problem (3.1) admits a $k_{\epsilon}-$ resolvent family $\left\{S_{\epsilon}(t)\right\}_{t \geq 0}$ in $X$ for each $\epsilon>0$ and that the stability condition (3.2) holds. Suppose also $a_{\epsilon}(t) \rightarrow a_{0}(t)$ and $k_{\epsilon}(t) \rightarrow k_{0}(t)$ as $\epsilon \rightarrow 0$. Then there is a $k_{0}$-regularized resolvent $\left\{S_{0}(t)\right\}_{t \geq 0}$ of type $(M, \omega)$ for the problem (3.1) with $\epsilon=0$ and

$$
S_{\epsilon}(t) x \rightarrow S_{0}(t) x
$$

as $\epsilon \rightarrow 0$ uniformly on compact subsets of $\mathbb{R}_{+} \times X$.

Throughout this paper, the symbol $*$ always denotes the finite convolution. We will follow the same notation as it is given in the monograph [10].

For $\epsilon>0$ we define:

$$
a_{\epsilon}(t)=1+(1 * K)(t)-e_{\epsilon}(t)-\left(e_{\epsilon} * K\right)(t), \quad t \geq 0,
$$

where $e_{\epsilon}(t):=e^{-\frac{1}{\epsilon^{2}} t}$ and for $\epsilon=0$

$$
a_{0}(t)=1+(1 * K)(t), \quad t \geq 0 .
$$

We always assume that the Laplace transform $\hat{a}_{\epsilon}(\lambda)$ exists and is non-zero for all $\lambda>\omega, \epsilon>0$ and some $\omega \in \mathbb{R}$. Then from (3.3) and (3.4) we easily see that

$$
\hat{a}_{\epsilon}(\lambda) \rightarrow \hat{a}_{0}(\lambda),
$$


as $\epsilon \rightarrow 0$ for all $\lambda$ sufficiently large.

In what follows we denote by $\mathcal{K}\left(\mathbb{R}, \epsilon_{0}\right)$ the set of all functions $K \in C^{1}(\mathbb{R})$ which satisfies the following conditions

1. $K(t) \geq 0$ for all $t>0$ and $K(0)>0$.

2. $K^{\prime}(t) \leq 0$ for all $t \geq 0$.

3. $\lim _{t \rightarrow \infty} K(t)=0$.

4. There exists $\epsilon_{0}>0$ such that for all $0<\epsilon \leq \epsilon_{0}$ the function

$$
\left(\frac{1}{\epsilon^{2}} K(t)+K^{\prime}(t)\right)\left(e_{\epsilon}(t)+\left(e_{\epsilon} * K\right)(t)\right)-K(t)^{2} \quad \text { is nonnegative. }
$$

Remark 3.2.

The above class $\mathcal{K}$ was introduced in [8]. A typical example is $K(t)=b e^{-a t}$ where $a>0, b>0$.

We recall that an infinitely differentiable function $f:(0, \infty) \rightarrow \mathbb{R}$ is called completely monotonic if

$$
(-1)^{n} f^{(n)}(\lambda) \geq 0
$$

for all $\lambda>0, n=0,1,2 \ldots$. The following result was proved in [8, Proposition 3.5].

Proposition 3.3. If $K \in \mathcal{K}\left(\mathbb{R}, \epsilon_{1}\right)$ then the function $h_{\epsilon}(\lambda, t):=\frac{1}{\lambda \sqrt{\hat{a}_{\epsilon}(\lambda)}} e^{-\frac{1}{\sqrt{\hat{a}_{\epsilon}(\lambda)}} t} \quad(t>0, \lambda>0)$ is completely monotonic in $\lambda$ for each $0<\epsilon<\epsilon_{1}$.

We state the following main result concerning the existence of resolvents satisfying the condition (3.2). Its proof follows the same reasoning as that of [9, Theorem 5] (see also, [10, Theorem 4.1]).

Theorem 3.4. Let $A$ be the generator of a 2-times integrated cosine function $\{S(t)\}_{t \geq 0}$ on a Banach space $X$ such that

$$
\|S(t)\| \leq M \cosh \left(w_{0} t\right), \quad t \geq 0,
$$

for some constants $M>0, w_{0} \in \mathbb{R}$. Assume $K \in \mathcal{K}\left(\mathbb{R}, \epsilon_{0}\right)$ is exponentially bounded. Then, the problem (3.1) admits a $\left(1 * a_{\epsilon}\right)$ - regularized family $\left\{S_{\epsilon}(t)\right\}_{t \geq 0}$ for all $0 \leq \epsilon<\epsilon_{0}$ such that for $x \in X$ $S_{\epsilon}(t) x \rightarrow S_{0}(t) x$ where the convergence is uniform on compact subsets of $\mathbb{R}_{+}$.

Proof. By definition, it follows from (3.7) that for all $x \in X,\left(w_{0}, \infty\right) \subset \rho(A)$ and

$$
(\lambda-A)^{-1} x=\sqrt{\lambda} \int_{0}^{+\infty} e^{-\sqrt{\lambda} t} S(t) x d t, \lambda>w_{0} .
$$

We claim that $A$ is the generator of an $k_{\epsilon}$-regularized family $S_{\epsilon}(t)$ for problem $(3.1)$, where $k_{\epsilon}(t):=$ $\left(1 * a_{\epsilon}\right)(t), t \geq 0$.

In fact, since $\hat{a}_{\epsilon}(\lambda) \rightarrow 0$ as $\lambda \rightarrow \infty$ we have that $\frac{1}{\hat{a}_{\epsilon}(\lambda)}>w_{0}$ for all $\lambda$ sufficiently large, say $\lambda>w_{1}$. We set for $x \in X$ and $\epsilon>0$,

$$
J_{\epsilon}(\lambda) x:=\frac{\widehat{k}_{\epsilon}(\lambda)}{\widehat{a}_{\epsilon}(\lambda)}\left[\frac{1}{\widehat{a}_{\epsilon}(\lambda)}-A\right]^{-1} x,
$$

whenever $\lambda>w_{1}$. Thus, for $x \in X$,

$$
\begin{aligned}
J_{\epsilon}(\lambda) x & =\frac{1}{\lambda \sqrt{\widehat{a}_{\epsilon}(\lambda)}} \int_{0}^{+\infty} e^{-\frac{1}{\sqrt{\hat{a}_{\epsilon}(\lambda)}} t} S(t) x d t \\
& =\int_{0}^{+\infty} h_{\epsilon}(\lambda, t) S(t) x d t .
\end{aligned}
$$

where $h_{\epsilon}(\lambda, t):=\frac{1}{\lambda \sqrt{\widehat{a}_{\epsilon}(\lambda)}} e^{-\frac{1}{\sqrt{\hat{a}_{\epsilon}(\lambda)}} t}, \lambda>w_{1}, t \geq 0$. 
Now, thanks to Proposition 3.3 we can now follow the same steps as those given in the proof of $[8$, Theorem 2.8]. Therefore there are constants $C>0$ and $w_{2} \in \mathbb{R}$ which are independent of $\epsilon>0$ such that

$$
\left\|L_{\lambda}^{n} J_{\epsilon}(\lambda)\right\| \leq \frac{C}{\left(\lambda-w_{2}\right)^{n+1}} \quad, n \in \mathbb{N} .
$$

Hence for all $0<\epsilon<\epsilon_{0}, A$ is the generator of an $1 * a_{\epsilon}$ - regularized resolvent family $S_{\epsilon}(t)$, by Theorem 2.3. Moreover,

$$
\left\|S_{\epsilon}(t)\right\| \leq C e^{w_{0} t}, t \geq 0
$$

Since $a_{\epsilon}(t) \rightarrow a_{0}(t)$ almost everywhere and $k_{\epsilon}(t)=\left(1 * a_{\epsilon}\right)(t) \rightarrow\left(1 * a_{0}\right)(t)=k_{0}(t)$ as $\epsilon \rightarrow 0^{+}$, we obtain from Theorem 3.1 that $A$ is the generator of an $\left(1 * a_{0}\right)$-regularized resolvent family $S_{0}(t)$, such that $\lim _{\epsilon \rightarrow 0^{+}} S_{\epsilon}(t) x=S_{0}(t) x$ for all $x \in X$ and $t \geq 0$. Moreover, the convergence in uniform on compact subsets of $\mathbb{R}_{+}$.

\section{Singular Perturbation}

Definition 4.1. We say that $u: \mathbb{R}_{+} \rightarrow X$ is a solution of (1.1) if $u \in C^{2}\left(\mathbb{R}_{+} ; X\right), u(t) \in D(A)$ for $t \geq 0$ and (1.1) is satisfied on $\mathbb{R}_{+}$.

Before proceeding to the main Theorem of this paper we state the next Lemma and some of its consequences.

In what follows, we denote $K^{*(1)}:=K$ and $K^{*(n)}:=K * K^{*(n-1)}$, for $n \geq 2$.

Lemma 4.2. Suppose that $K(t)$ is continuous and exponentially bounded and define

$$
r_{\epsilon}(t):=\epsilon^{2}\left(1+\sum_{n \geq 1}(-1)^{n}\left(1 * K^{*(n)}\right)\right)(t)+t+\sum_{n \geq 1}(-1)^{n}\left(t * K^{*(n)}\right)(t),
$$

for $\epsilon \geq 0, t \geq 0$. Then,

(i) The series (4.1) is absolutely convergent for $t \geq 0$ and uniformly convergent on compact intervals. Moreover, there is $M \geq 1, w \in \mathbb{R}_{+}$such that

$$
\left|r_{\epsilon}(t)\right| \leq M e^{w t} \quad, \quad t \geq 0 .
$$

(ii) Let $r_{0}(t):=t+\sum_{n \geq 1}(-1)^{n}\left(t * K^{*(n)}\right)(t), t \geq 0$ then $\lim _{\epsilon \rightarrow 0^{+}} r_{\epsilon}(t)=r_{0}(t)$ uniformly in $t \geq 0$.

(iii) For $\epsilon \geq 0, r_{\epsilon}(t)$ satisfy

$$
\begin{gathered}
\left(r_{\epsilon} * a_{\epsilon}\right)(t)=\frac{t^{2}}{2} \quad, \quad t \geq 0 . \\
\left(r_{\epsilon} * a_{0}\right)(t)=\frac{t^{2}}{2}+\epsilon^{2} t \quad, \quad t \geq 0 .
\end{gathered}
$$

(iv) For each $\epsilon \geq 0, r_{\epsilon} \in C^{1}\left(\mathbb{R}_{+}\right)$and

$$
\epsilon^{2} a_{0}(t)+\left(r_{\epsilon}^{\prime} * a_{0}\right)(t)=t+\epsilon^{2},
$$

in particular for $\epsilon=0$

$$
\left(r_{0}^{\prime} * a_{0}\right)(t)=t
$$


Proof. Since $K(t)$ es exponentially bounded, there is $M>0$ and $w \geq 0$ such that $\|K(t)\| \leq M e^{w t}$. Then,

$$
|(1 * K)(t)| \leq M\left(e^{w t} * e^{w t}\right)(t)=M t e^{w t}
$$

and inductively,

Thus

$$
\left|\left(1 * K^{*(n)}\right)(t)\right| \leq \frac{M^{n}}{n !} t^{n} e^{w t} \quad \text { for } n=1,2, \ldots
$$

$$
1+\sum_{n \geq 1}\left|\left(1 * K^{*(n)}\right)(t)\right| \leq e^{w t}+e^{w t} \sum_{n \geq 1} \frac{M^{n} t^{n}}{n !}=e^{(w+M) t} .
$$

On the other hand

$$
|(t * K)(t)| \leq M\left(e^{w t} * e^{w t}\right)(t)=M t e^{w t}
$$

and

Thus

$$
\left|\left(t * K^{*(n)}\right)(t)\right| \leq \frac{M^{n}}{n !} t^{n} e^{w t} \text { for } n=1,2, \ldots
$$

$$
t+\sum_{n \geq 1}\left|\left(t * K^{*(n)}\right)(t)\right| \leq e^{(w+M) t},
$$

and hence $r_{\epsilon}(t)$ is exponentially bounded and then claim (i) follows. The proof of (ii) is immediate. To show (iii) notice that $\widehat{r}_{\epsilon}(\lambda)=\frac{\epsilon^{2}}{1+\widehat{K}(\lambda)}+\frac{1}{\lambda^{2}(1+\widehat{K}(\lambda))}, \lambda>w$ and $\widehat{a}_{\epsilon}(\lambda)=\frac{1+\widehat{K}(\lambda)}{\lambda^{2} \epsilon^{2}+\lambda}, \lambda>w$. Then $\widehat{r}_{\epsilon}(\lambda) \widehat{a}_{\epsilon}(\lambda)=\frac{1}{\lambda^{3}}$ and the proof of (4.2) follows by uniqueness of the Laplace transform, and by the same reasoning we show (4.3). The claim (iv) is obvious.

\section{Remark 4.3.}

A direct consequence of the above Lemma are the following identities, which we will be needed in the proof of Theorem 3.6 below.

$$
\begin{aligned}
& \left(r_{\epsilon}^{\prime} * a_{\epsilon}\right)(t)=t-\epsilon^{2} a_{\epsilon}(t) \\
& \left(r_{\epsilon}^{\prime} * a_{\epsilon}^{\prime}\right)(t)=1-\epsilon^{2} a_{\epsilon}^{\prime}(t) \\
& \left(r_{\epsilon}^{\prime} * a_{\epsilon}^{\prime \prime}\right)(t)=-\epsilon^{2} a_{\epsilon}^{\prime \prime}(t)-\frac{r_{\epsilon}^{\prime}(t)}{\epsilon^{2}} \\
& \left(r_{0} * a_{0}\right)(t)=\frac{t^{2}}{2} \\
& r_{0}(t)+\left(r_{0}^{\prime} * K\right)(t)=1 \\
& r_{0}^{\prime \prime}(t)+K(t)+\left(r_{0}^{\prime \prime} * K\right)(t)=0 \\
& r_{\epsilon}^{\prime}(t)+\left(K * r_{\epsilon}^{\prime}\right)(t)+\epsilon^{2} K=1
\end{aligned}
$$

Proof. To show (A1), we notice that $\left(r_{\epsilon} * a_{\epsilon}\right)(t)=t^{2} / 2$ by equation $(4.2)$, then $\left(r_{\epsilon} * a_{\epsilon}\right)^{\prime}(t)=t$ and since $r_{\epsilon}(0)=\epsilon^{2}$ it then follows that $\left(r_{\epsilon}^{\prime} * a_{\epsilon}\right)(t)=t-\epsilon^{2} a_{\epsilon}(t)$. Now from (A1), we get that $\left(r_{\epsilon}^{\prime} * a_{\epsilon}\right)^{\prime}(t)=1-\epsilon^{2} a_{\epsilon}^{\prime}(t)$ and since $a_{\epsilon}(0)=0$ we obtain (A2). In order to prove (A3), we recall that $a_{\epsilon}^{\prime}(t)=\frac{1}{\epsilon^{2}} e_{\epsilon}(t)+\frac{1}{\epsilon^{2}}\left(e_{\epsilon} * K\right)(t)$ and hence $a_{\epsilon}^{\prime}(0)=1 / \epsilon^{2}$, and now the proof follows by differentiation on both sides of (A2). To show (A4) we recall that $\left(r_{0}^{\prime} * a_{0}\right)(t)=t$ by equation (4.5) and since $r_{0}(0)=0$ then (A4) follows by the fact that $\left(1 * r_{0}^{\prime} * a_{0}\right)(t)=1 * t=t^{2} / 2$. The proof of (A5),(A6) uses (4.5) and (A7)uses (4.4) and the fact that $a_{0}^{\prime}(t)=K(t)$ and then we apply the same reasoning as when we show the previous identities. 
Remark 4.4 .

In the proof of the next Theorem we also will make use some properties concerning the behavior under convolution of the function $e_{\epsilon}(t)=e^{-\frac{1}{\epsilon^{2}} t}$ as $\epsilon \rightarrow 0^{+}$. That is, given a scalar valued function $f$ belonging to $C\left(\mathbb{R}_{+}\right)$, and $t \geq 0$, the following properties are true:

$$
\begin{aligned}
& \left|\left(e_{\epsilon} * f\right)(t)\right| \leq t \sup \{|f(s)|: 0 \leq s \leq t\}, \quad \text { for all } \epsilon>0, \\
& \lim _{\epsilon \rightarrow 0^{+}}\left(e_{\epsilon} * f\right)(t)=0,
\end{aligned}
$$

Before to proceed to the following theorem observe that a calculation yields

$$
a_{\epsilon}^{\prime}(t)=\frac{1}{\epsilon^{2}} e_{\epsilon}(t)+\frac{1}{\epsilon^{2}}\left(e_{\epsilon} * K\right)(t) .
$$

and

$$
a_{\epsilon}^{\prime \prime}(t)=\left(\frac{K(0)}{\epsilon^{2}}-\frac{1}{\epsilon^{4}}\right) e_{\epsilon}(t)+\frac{1}{\epsilon^{2}}\left(e_{\epsilon} * K^{\prime}\right)(t) .
$$

We are now ready to state and proof the main theorem of this paper.

Theorem 4.5. Let $A$ be the generator of a 2-times integrated cosine family on a Banach space $X$. Suppose that $K \in \mathcal{K}\left(\mathbb{R}, \epsilon_{0}\right)$ is exponentially bounded, and

(H1) $u_{0}(\epsilon), u_{1}(\epsilon), w_{0} \in D(A), u_{0}(\epsilon) \rightarrow w_{0}$ and $u_{1}(\epsilon) \rightarrow w_{1} \in X$, as $\epsilon \rightarrow 0^{+}$.

(H2) $A u_{0}(\epsilon) \rightarrow 0$ and there is $C \geq 0$ such that $\left\|A u_{1}(\epsilon)\right\| \leq C$ for every $\epsilon>0$.

(H3) $f_{\epsilon}(\cdot) \in C^{1}\left(\mathbb{R}_{+} ; D(A)\right)$, and as $\epsilon \rightarrow 0^{+} f_{\epsilon}(t) \rightarrow f(t)$ point-wise and $f_{\epsilon}^{\prime}(\cdot) \rightarrow f^{\prime}(\cdot)$ in $L^{1}([0, b], D(A))$ for every $b \geq 0$.

Then problem (1.1) has a unique solution $u_{\epsilon}(t)$ for all $0<\epsilon<\epsilon_{0}$ which converges to the unique solution $u(t)$ of problem (1.2). Moreover the convergence is uniform on compact intervals of $\mathbb{R}_{+}$.

Proof We claim that the solution $u_{\epsilon}(t), \epsilon>0$ of equation (1.1) can be represented by means of the $\left(1 * a_{\epsilon}\right)$-regularized resolvent family $S_{\epsilon}(t)$ given in Theorem 3.1.

We note first that since $K$ is continuous then $a_{\epsilon} \in C^{2}([0,+\infty))$ and from Remark 2.2 follows

$$
S_{\epsilon}(t) x=\left(1 * a_{\epsilon}\right)(t) x+\left(a_{\epsilon} * A S_{\epsilon}\right)(t) x, \quad x \in D(A) ;
$$

thus, for $u_{0} \in D(A)$, and $\epsilon \geq 0 S_{\epsilon}(t) u_{0} \in C^{2}([0,+\infty) ; X)$. Hence from equation (4.8) and since $a_{\epsilon}(0)=0$ it follows

$$
S_{\epsilon}^{\prime}(t) u_{0}=a_{\epsilon}(t) u_{0}+\left(a_{\epsilon}^{\prime} * A S_{\epsilon}\right)(t) u_{0} .
$$

Furthermore we have that

$$
S_{\epsilon}^{\prime \prime}(t) u_{0}=a_{\epsilon}^{\prime}(t) u_{0}+\frac{1}{\epsilon^{2}} A S_{\epsilon}(t) u_{0}+\left(a_{\epsilon}^{\prime \prime} * A S_{\epsilon}\right)(t) u_{0},
$$

since $a_{\epsilon}^{\prime}(0)=\frac{1}{\epsilon^{2}}$ by (4.6). Next we define for each $\epsilon>0, t \geq 0$,

$$
u_{\epsilon}(t):=\left(r_{\epsilon}^{\prime} * S_{\epsilon}^{\prime \prime}\right)(t) u_{0}+\epsilon^{2} S_{\epsilon}^{\prime \prime}(t) u_{0}+\epsilon^{2}\left(r_{0}^{\prime} * S_{\epsilon}^{\prime \prime}\right)(t) u_{1}+\left(r_{0}^{\prime} * S_{\epsilon}^{\prime \prime} * f_{\epsilon}\right)(t)
$$

where $u_{0}:=u_{0}(\epsilon), u_{1}:=u_{1}(\epsilon)$. We claim that $u_{\epsilon}(t)$ satisfy the integrated equation

$$
\epsilon^{2}\left(u_{\epsilon}(t)-u_{0}-u_{1} t\right)+\left(1 * u_{\epsilon}\right)(t)-t u_{0}=A\left(t * u_{\epsilon}\right)(t)+\left(K * A\left(t * u_{\epsilon}\right)\right)(t)+\left(t * f_{\epsilon}\right)(t) .
$$

Towards this end we define,

$$
\begin{array}{llll}
L_{1}(t):=\left(r_{\epsilon}^{\prime} * S_{\epsilon}^{\prime \prime}\right)(t) u_{0} & , & L_{2}(t):=S_{\epsilon}^{\prime \prime}(t) u_{0} \\
L_{3}(t):=\left(r_{0}^{\prime} * S_{\epsilon}^{\prime \prime}\right)(t) u_{1} & , & L_{4}(t):=\left(r_{0}^{\prime} * S_{\epsilon}^{\prime \prime} * f_{\epsilon}\right)(t) .
\end{array}
$$


Then applying (4.10), we get that

$$
\begin{aligned}
L_{1}(t) & =r_{\epsilon}^{\prime} *\left[a_{\epsilon}^{\prime}+\frac{1}{\epsilon^{2}} A S_{\epsilon}+a_{\epsilon}^{\prime \prime} * A S_{\epsilon}\right](t) u_{0} \\
& =\left[r_{\epsilon}^{\prime} * a_{\epsilon}^{\prime}+r_{\epsilon}^{\prime} * \frac{1}{\epsilon^{2}} A S_{\epsilon}-\left(\epsilon^{2} a_{\epsilon}^{\prime \prime}+\frac{r_{\epsilon}^{\prime}}{\epsilon^{2}}\right) * A S_{\epsilon}\right](t) u_{0}
\end{aligned}
$$

since $\left(r_{\epsilon}^{\prime} * a_{\epsilon}^{\prime}\right)(t)=1-\epsilon^{2} a_{\epsilon}^{\prime}(t)$ and $\left(r_{\epsilon}^{\prime} * a_{\epsilon}^{\prime \prime}\right)(t)=-\epsilon^{2} a_{\epsilon}^{\prime \prime}(t)-\frac{r_{\epsilon}^{\prime}(t)}{\epsilon^{2}}$, by (A2) and (A3) of Remark 4.3. Thus

$$
L_{1}(t)=u_{0}-\epsilon^{2} a_{\epsilon}^{\prime}(t) u_{0}-\epsilon^{2}\left(a_{\epsilon}^{\prime \prime} * A S_{\epsilon}\right)(t) u_{0} .
$$

On the other hand $a_{\epsilon}^{\prime}(t)=-\frac{s_{\epsilon}(t)}{\epsilon^{2}}$ and $a_{\epsilon}^{\prime \prime}(t)=-\frac{s_{\epsilon}^{\prime}(t)}{\epsilon^{2}}$, where

$$
s_{\epsilon}(t):=-e_{\epsilon}(t)-\left(e_{\epsilon} * K\right)(t) .
$$

Thus,

$$
L_{3}(t)=\left(r_{0}^{\prime} * S_{\epsilon}^{\prime \prime}\right)(t) u_{1}=\left(-r_{0}^{\prime} * \frac{s_{\epsilon}}{\epsilon^{2}}+r_{0}^{\prime} * \frac{A S_{\epsilon}}{\epsilon^{2}}-r_{0}^{\prime} * \frac{s_{\epsilon}^{\prime}}{\epsilon^{2}} * A S_{\epsilon}\right)(t) u_{1}
$$

and

$$
\begin{aligned}
L_{4}(t) & =\left(r_{0}^{\prime} * S_{\epsilon}^{\prime \prime} * f_{\epsilon}\right)(t) \\
& =\left(-r_{0}^{\prime} * \frac{s_{\epsilon}}{\epsilon^{2}} * f_{\epsilon}\right)(t)+\left(r_{0}^{\prime} * \frac{A S_{\epsilon}}{\epsilon^{2}} * f_{\epsilon}\right)(t)-\left(r_{0}^{\prime} * \frac{s_{\epsilon}^{\prime}}{\epsilon^{2}} * A S_{\epsilon} * f_{\epsilon}\right)(t) .
\end{aligned}
$$

Hence

$$
\begin{aligned}
\epsilon^{2}\left(u_{\epsilon}(t)-u_{0}-u_{1} t\right) & =\epsilon^{2} L_{1}(t)+\epsilon^{4} L_{2}(t)+\epsilon^{4} L_{3}(t)+\epsilon^{2} L_{4}(t)-\epsilon^{2} u_{0}-\epsilon^{2} u_{1} t \\
& =\epsilon^{2} A S_{\epsilon}(t) u_{0}-\epsilon^{2}\left(r_{0}^{\prime} * s_{\epsilon}\right)(t) u_{1}+\epsilon^{2}\left(r_{0}^{\prime} * A S_{\epsilon}\right)(t) u_{1} \\
& -\epsilon^{2}\left(r_{0}^{\prime} * s_{\epsilon}^{\prime} * A S_{\epsilon}\right)(t) u_{1}-\left(r_{0}^{\prime} * s_{\epsilon} * f_{\epsilon}\right)(t)+\left(r_{0}^{\prime} * A S_{\epsilon} * f_{\epsilon}\right)(t) \\
& -\left(r_{0}^{\prime} * s_{\epsilon}^{\prime} * A S_{\epsilon} * f_{\epsilon}\right)(t)-\epsilon^{2} u_{1} t
\end{aligned}
$$

Since $S_{\epsilon}^{\prime}(0)=0$, we obtain that

$$
\left(1 * u_{\epsilon}\right)(t)=\left(r_{\epsilon}^{\prime} * S_{\epsilon}^{\prime}\right)(t) u_{0}+\epsilon^{2} S_{\epsilon}^{\prime}(t) u_{0}+\epsilon^{2}\left(r_{0}^{\prime} * S_{\epsilon}^{\prime}\right)(t) u_{1}+\left(r_{0}^{\prime} * S_{\epsilon}^{\prime} * f_{\epsilon}\right)(t)
$$

We now recall that $\left(r_{\epsilon}^{\prime} * a_{\epsilon}\right)(t)=t-\epsilon^{2} a_{\epsilon}(t)$ and $\left(r_{\epsilon}^{\prime} * a_{\epsilon}^{\prime}\right)(t)=1-\epsilon^{2} a_{\epsilon}^{\prime}(t)$ by equations (A1) and (A2) respectively, thus we have that

$$
\begin{aligned}
\left(r_{\epsilon}^{\prime} * S_{\epsilon}^{\prime}\right)(t) u_{0} & =\left(r_{\epsilon}^{\prime} * a_{\epsilon}\right)(t) u_{0}+\left(r_{\epsilon}^{\prime} * a_{\epsilon}^{\prime} * A S_{\epsilon}\right)(t) u_{0} \\
& =t u_{0}-\epsilon^{2} a_{\epsilon}(t) u_{0}+\left(\left(1-\epsilon^{2} a_{\epsilon}^{\prime}\right) * A S_{\epsilon}\right)(t) u_{0} \\
& =t u_{0}+\left(1 * A S_{\epsilon}\right)(t) u_{0}-\epsilon^{2} a_{\epsilon}(t) u_{0}-\epsilon^{2}\left(a_{\epsilon}^{\prime} * A S_{\epsilon}\right)(t) u_{0}
\end{aligned}
$$

and hence

$$
\begin{aligned}
\left(1 * u_{\epsilon}\right)(t) & =t u_{0}+\left(1 * A S_{\epsilon}\right)(t) u_{0}-\epsilon^{2} a_{\epsilon}(t) u_{0}-\epsilon^{2}\left(a_{\epsilon}^{\prime} * A S_{\epsilon}\right)(t) u_{0} \\
& +\epsilon^{2} a_{\epsilon}(t) u_{0}+\epsilon^{2}\left(a_{\epsilon}^{\prime} * A S_{\epsilon}\right)(t) u_{0}+\epsilon^{2}\left(r_{0}^{\prime} * a\right)(t) u_{1} \\
& +\epsilon^{2}\left(r_{0}^{\prime} * s_{\epsilon}\right)(t) u_{1}-\left(r_{0}^{\prime} * s_{\epsilon} * A S_{\epsilon}\right)(t) u_{1} \\
& +\left(r_{0}^{\prime} * a * f_{\epsilon}\right)(t)+\left(r_{0}^{\prime} * s_{\epsilon} * f_{\epsilon}\right)(t)+\left(r_{0}^{\prime} * a_{\epsilon}^{\prime} * A S_{\epsilon} * f_{\epsilon}\right)(t)
\end{aligned}
$$


From the fact that $\epsilon^{2} s_{\epsilon}^{\prime}(t)+s_{\epsilon}(t)=-K(t)$ and $a_{\epsilon}^{\prime}(t)-s_{\epsilon}^{\prime}(t)=K(t)$, we obtain that

$$
\begin{aligned}
V_{\epsilon}(t) & :=\epsilon^{2}\left(u_{\epsilon}(t)-u_{0}-u_{1} t\right)+\left(1 * u_{\epsilon}\right)(t)-t u_{0} \\
& =\epsilon^{2} A S_{\epsilon}(t) u_{0}+\left(1 * A S_{\epsilon}\right)(t) u_{0}+\epsilon^{2}\left(r_{0}^{\prime} * A S_{\epsilon}\right)(t) u_{1} \\
& +\epsilon^{2}\left(r_{0}^{\prime} * K * A S_{\epsilon}\right)(t) u_{1}+\left(\left(r_{0}^{\prime}+r_{0}^{\prime} *\left(a_{\epsilon}^{\prime}-s_{\epsilon}^{\prime}\right)\right) * A S_{\epsilon} * f_{\epsilon}\right)(t)+\left(t * f_{\epsilon}\right)(t) \\
& =\epsilon^{2} A S_{\epsilon}(t) u_{0}+\left(1 * A S_{\epsilon}\right)(t) u_{0}+\epsilon^{2}\left(r_{0}^{\prime} * A S_{\epsilon}\right)(t) u_{1} \\
& +\epsilon^{2}\left(r_{0}^{\prime} * K * A S_{\epsilon}\right)(t) u_{1}+\left(\left(r_{0}^{\prime}+r_{0}^{\prime} * K\right) * A S_{\epsilon} * f_{\epsilon}\right)(t)+\left(t * f_{\epsilon}\right)(t) \\
& =\epsilon^{2} A S_{\epsilon}(t) u_{0}+\left(1 * A S_{\epsilon}\right)(t) u_{0}+\epsilon^{2}\left(\left(r_{0}^{\prime}+r_{0}^{\prime} * K\right) * A S_{\epsilon}\right)(t) u_{1} \\
& +\left(\left(r_{0}^{\prime}+r_{0}^{\prime} * K\right) * A S_{\epsilon} * f_{\epsilon}\right)(t)+\left(t * f_{\epsilon}\right)(t) .
\end{aligned}
$$

On the other hand we have that

$$
\begin{aligned}
W_{\epsilon}(t) & :=A\left(t * u_{\epsilon}\right)(t)+\left(K * A\left(t * u_{\epsilon}\right)\right)(t)+\left(t * f_{\epsilon}\right)(t) \\
& =\left(r_{\epsilon}^{\prime} * A S_{\epsilon}\right)(t) u_{0}+\epsilon^{2} A S_{\epsilon}(t) u_{0}+\left(K * r_{\epsilon}^{\prime} * A S_{\epsilon}\right)(t) u_{0} \\
& +\epsilon^{2}\left(K * A S_{\epsilon}\right)(t) u_{0}+\epsilon^{2}\left(r_{0}^{\prime} * A S_{\epsilon}\right)(t) u_{1}+\epsilon^{2}\left(K * r_{0}^{\prime} * A S_{\epsilon}\right)(t) u_{1} \\
& +\left(\left(r_{0}^{\prime}+r_{0}^{\prime} * K\right) * A S_{\epsilon} * f_{\epsilon}\right)(t)+\left(t * f_{\epsilon}\right)(t) \\
& =\left(\left(r_{\epsilon}^{\prime}+K * r_{\epsilon}^{\prime}+\epsilon^{2} K\right) * A S_{\epsilon}\right)(t) u_{0}+\epsilon^{2} A S_{\epsilon}(t) u_{0} \\
& +\epsilon^{2}\left(\left(r_{0}^{\prime}+r_{0}^{\prime} * K\right) * A S_{\epsilon}\right)(t) u_{1}+\left(\left(r_{0}^{\prime}+r_{0}^{\prime} * K\right) * A S_{\epsilon} * f_{\epsilon}\right)(t)+\left(t * f_{\epsilon}\right)(t),
\end{aligned}
$$

and since $r_{\epsilon}^{\prime}(t)+\left(K * r_{\epsilon}^{\prime}\right)(t)+\epsilon^{2} K(t)=1$ by $(\mathrm{A} 7)$ we get that $V_{\epsilon}(t)=W_{\epsilon}(t)$. Thus we have proved that $u_{\epsilon}(t)$ satisfy equation (1.1) and it can be represented by (4.11).

Now when $u_{0}=u(\epsilon)$ in (4.11) we show that $u_{\epsilon}(t)$ converges towards

$$
u(t):=w_{0}+\left(S_{0}^{\prime} * f\right)(t)+\left(r_{0}^{\prime \prime} * S_{0}^{\prime} * f\right)(t)
$$

as $\epsilon \rightarrow 0^{+}$, where $w_{0}=\lim _{\epsilon \rightarrow 0^{+}} u_{0}(\epsilon)$. We recall that $\lim _{\epsilon \rightarrow 0^{+}} S_{\epsilon}(t) u_{\epsilon}(0)=S_{0}(t) w_{0}$ by Theorem 3.1. On the other hand

$$
u_{\epsilon}(t)=\left(r_{\epsilon}^{\prime} * S_{\epsilon}^{\prime \prime}\right)(t) u_{0}(\epsilon)+\epsilon^{2} S_{\epsilon}^{\prime \prime}(t) u_{0}(\epsilon)+\epsilon^{2}\left(r_{0}^{\prime} * S_{\epsilon}^{\prime \prime}\right)(t) u_{1}(\epsilon)+\left(r_{0}^{\prime} * S_{\epsilon}^{\prime \prime} * f_{\epsilon}\right)(t)
$$

by equation (4.11); now, for this representation of $u_{\epsilon}(t)$ we are going to compute the limit of each term of the sum separately, and that the convergence is uniform on an interval $I_{b}:=[0, b]$. First we notice that

$$
\left(r_{\epsilon}^{\prime} * S_{\epsilon}^{\prime \prime}\right)(t) u_{0}(\epsilon)=u_{0}(\epsilon)-\epsilon^{2} a_{\epsilon}^{\prime}(t) u_{0}(\epsilon)-\epsilon^{2}\left(a_{\epsilon}^{\prime \prime} * A S_{\epsilon}\right)(t) u_{0}(\epsilon)
$$

by equation (4.12). We claim that

$$
\lim _{\epsilon \rightarrow 0^{+}}\left(r_{\epsilon}^{\prime} * S_{\epsilon}^{\prime \prime}\right)(t) u_{0}(\epsilon)=w_{0} .
$$

Since $\epsilon^{2} a_{\epsilon}^{\prime}(t) \leq \epsilon^{2}\left(1+t\|K\|_{\infty}\right)$ by (B1). Thus $\lim _{\epsilon \rightarrow 0+} \epsilon^{2} a_{\epsilon}^{\prime}(t)=0$ uniformly for $t \in I_{b}$. Hence it suffices to show that

$$
\lim _{\epsilon \rightarrow 0+} \epsilon^{2}\left(a_{\epsilon}^{\prime \prime} * A S_{\epsilon}\right)(t) u_{0}(\epsilon)=0 .
$$

To this end, we recall that $a_{\epsilon}^{\prime \prime}(t) \leq 0$ and $a_{\epsilon}^{\prime}(t) \geq 0$ thus $\epsilon^{2} \int_{0}^{b}\left|a_{\epsilon}^{\prime \prime}(s)\right| d s=1-\epsilon^{2} a_{\epsilon}^{\prime}(b) \leq 1$. Moreover

$$
\begin{aligned}
\left\|\epsilon^{2}\left(a_{\epsilon}^{\prime \prime} * A S_{\epsilon}\right)(t) u_{0}(\epsilon)\right\| & \leq \epsilon^{2}\left\|A u_{0}(\epsilon)\right\| \int_{0}^{b}\left|a_{\epsilon}^{\prime \prime}(s)\right|\left\|S_{\epsilon}(b-s)\right\| d s \\
& \leq M e^{\omega b}\left\|A u_{0}(\epsilon)\right\| \epsilon^{2} \int_{0}^{b}\left|a_{\epsilon}^{\prime \prime}(s)\right| d s \\
& \leq M e^{\omega b}\left\|A u_{0}(\epsilon)\right\| .
\end{aligned}
$$

for $t \in I_{b}$. Thus the limit (4.14) follows by assumption (H2). 
We now show that

$$
\lim _{\epsilon \rightarrow 0^{+}} \epsilon^{2} S_{\epsilon}^{\prime \prime}(t) u_{0}(\epsilon)=0
$$

Since we have already proved that $\lim _{\epsilon \rightarrow 0+} \epsilon^{2}\left(a_{\epsilon}^{\prime \prime} * A S_{\epsilon}\right)(t) u_{0}(\epsilon)=0$. Then by considering the fact that

$$
\epsilon^{2} S_{\epsilon}^{\prime \prime}(t) u_{0}(\epsilon)=\epsilon^{2} a_{\epsilon}^{\prime}(t) u_{0}(\epsilon)+A S_{\epsilon}(t) u_{0}(\epsilon)+\epsilon^{2}\left(a_{\epsilon}^{\prime \prime} * A S_{\epsilon}\right)(t) u_{0}(\epsilon),
$$

and since $\epsilon^{2} a_{\epsilon}^{\prime}(t) u_{0}(\epsilon)$ tends to 0 , the proof of (4.15) follows.

Next we show that

$$
\lim _{\epsilon \rightarrow 0^{+}} \epsilon^{2}\left(r_{0}^{\prime} * S_{\epsilon}^{\prime \prime}\right)(t) u_{1}(\epsilon)=0
$$

Since $S_{\epsilon}^{\prime}(0)=0$ and $r_{0}^{\prime}(0)=1$ it follows that $\left(r_{0}^{\prime} * S_{\epsilon}^{\prime \prime}\right)(t)=S_{\epsilon}^{\prime}(t)+\left(r_{0}^{\prime \prime} * S_{\epsilon}^{\prime}\right)(t)$, then we remark that

$$
\left\|\epsilon^{2} S_{\epsilon}^{\prime}(t) u_{1}(\epsilon)\right\| \leq\left|\epsilon^{2} a_{\epsilon}(t)\right|\left\|u_{1}(\epsilon)\right\|+\left\|\epsilon^{2}\left(a_{\epsilon}^{\prime} * S_{\epsilon}\right)(t) A u_{1}(\epsilon)\right\|,
$$

and we notice that

$$
\left\|\epsilon^{2}\left(a_{\epsilon}^{\prime} * S_{\epsilon}\right)(t) A u_{1}(\epsilon)\right\| \leq M e^{\omega b}\left\|A u_{1}(\epsilon)\right\| \epsilon^{2} \int_{0}^{b}\left|a_{\epsilon}^{\prime}(s)\right| d s
$$

by the stability condition (3.2), and since this last integral and the sequence of functions $\left|\epsilon^{2} a_{\epsilon}(t)\right|\left\|u_{1}(\epsilon)\right\|$ both converge to 0 uniformly for $t \in I_{b}$, it then follows that $\left\|\epsilon^{2} S_{\epsilon}^{\prime}(t) u_{1}(\epsilon)\right\| \rightarrow 0$ uniformly for $t \in[0, b]$, hence $\left\|r_{0}^{\prime \prime}\right\|_{\infty} \int_{0}^{b} \epsilon^{2}\left\|S_{\epsilon}^{\prime}(s) u_{1}(\epsilon)\right\| d s \rightarrow 0$. On the other hand for $t \in I_{b}$ we have that,

$$
\left\|\epsilon^{2}\left(r_{0}^{\prime \prime} * S_{\epsilon}^{\prime}\right)(t) u_{1}(\epsilon)\right\| \leq\left\|r_{0}^{\prime \prime}\right\|_{\infty} \int_{0}^{b} \epsilon^{2}\left\|S_{\epsilon}^{\prime}(s) u_{1}(\epsilon)\right\| d s,
$$

and hence the proof of (4.16) follows.

Next we claim that

$$
\lim _{\epsilon \rightarrow 0+}\left(r_{0}^{\prime} * S_{\epsilon}^{\prime \prime} * f_{\epsilon}\right)(t)=\left(r_{0}^{\prime \prime} * S_{0}^{\prime} * f\right)(t)+\left(S_{0}^{\prime} * f\right)(t)
$$

To this end we notice that we can write

$$
\left(r_{0}^{\prime} * S_{\epsilon}^{\prime \prime} * f_{\epsilon}\right)(t)=\left(S_{\epsilon}^{\prime} * f_{\epsilon}\right)(t)+\left(r_{0}^{\prime \prime} * S_{\epsilon}^{\prime} * f_{\epsilon}\right)(t) .
$$

Moreover,

$$
\left(S_{\epsilon}^{\prime} * f_{\epsilon}\right)(t)=S_{\epsilon}(t)\left(f_{\epsilon}(0)\right)+\left(S_{\epsilon} * f_{\epsilon}^{\prime}\right)(t) .
$$

Now in order to compute the limit of $\left(S_{\epsilon} * f_{\epsilon}^{\prime}\right)(t)$ as $\epsilon \rightarrow 0^{+}$, we recall that $f_{\epsilon}^{\prime} \rightarrow f^{\prime}$ in $L^{1}([0, b] ; D(A))$, by (H3). Hence we obtain that

$$
\int_{0}^{t} \| S_{\epsilon}(t-s)\left(f_{\epsilon}^{\prime}(s)-f^{\prime}(s)\left\|d s \leq M e^{\omega b} \int_{0}^{b}\right\| f_{\epsilon}^{\prime}(s)-f^{\prime}(s) \| d s \rightarrow 0,\right.
$$

as $\epsilon \rightarrow 0^{+}$. Moreover, for $t \in[0, b]$

$$
\int_{0}^{t}\left\|\left(S_{\epsilon}(t-s)-S_{0}(t-s)\right)\left(f^{\prime}(s)\right)\right\| d s \leq b \sup _{0 \leq s \leq b}\left\|\left(S_{\epsilon}(s)-S_{0}(s)\right)\left(f^{\prime}(t-s)\right)\right\| .
$$

Since $S_{\epsilon}(t)\left(f^{\prime}(t)\right) \rightarrow S_{0}(t)\left(f^{\prime}(t)\right)$ as $\epsilon \rightarrow 0^{+}$uniformly for $t \in[0, b]$ by Theorem 3.1, implying that $\sup _{0 \leq s \leq b}\left\|\left(S_{\epsilon}(s)-S_{0}(s)\right)\left(f^{\prime}(s)\right)\right\| \rightarrow 0$ and hence

$$
\int_{0}^{t}\left\|\left(S_{\epsilon}(t-s)-S_{0}(t-s)\right)\left(f^{\prime}(s)\right)\right\| d s \rightarrow 0
$$


as $\epsilon \rightarrow 0^{+}$, uniformly for $t \in I_{b}$. Thus from the triangle inequality we have that

$$
\lim _{\epsilon \rightarrow 0^{+}}\left(S_{\epsilon} * f_{\epsilon}^{\prime}\right)(t)=\left(S_{0} * f^{\prime}\right)(t)
$$

by applying the estimates (4.19) and (4.20). Hence we have shown that

$$
\begin{aligned}
\lim _{\epsilon \rightarrow 0^{+}}\left(S_{\epsilon}^{\prime} * f_{\epsilon}\right)(t) & =\lim _{\epsilon \rightarrow 0^{+}}\left(S_{\epsilon}(t)\left(f_{\epsilon}(0)\right)+\left(S_{\epsilon} * f_{\epsilon}^{\prime}\right)(t)\right. \\
& =S_{0}(t)(f(0))+\left(S_{0} * f^{\prime}\right)(t) .
\end{aligned}
$$

Next, we calculate the limit of $\left(r_{0}^{\prime \prime} * S_{\epsilon}^{\prime} * f_{\epsilon}\right)(t)$ as $\epsilon \rightarrow 0^{+}$. First we notice that

$$
\left(r_{0}^{\prime \prime} * S_{\epsilon}^{\prime} * f_{\epsilon}\right)(t)=\left(r_{0}^{\prime \prime} * S_{\epsilon}(t)\right)\left(f_{\epsilon}(0)\right)+\left(r_{0}^{\prime \prime} * S_{\epsilon} * f_{\epsilon}^{\prime}\right)(t) .
$$

Since $\left(r_{0}^{\prime \prime} * S_{\epsilon}(t)\right)\left(f_{\epsilon}(0)\right) \rightarrow\left(r_{0}^{\prime \prime} * S_{0}\right)(t)\left(f(0)\right.$, we only have to compute the limit of $\left(r_{0}^{\prime \prime} * S_{\epsilon} * f_{\epsilon}^{\prime}\right)(t)$. Hence we define $A_{\epsilon}(t):=\int_{0}^{t}\left\|\left(S_{\epsilon} *\left(f_{\epsilon}^{\prime}-f^{\prime}\right)\right)(s)\right\| d s$ and $B_{\epsilon}(t):=\int_{0}^{t}\left\|\left(\left(S_{\epsilon}-S_{0}\right) * f^{\prime}\right)(s)\right\| d s$. Then

$$
\left\|\left(r_{0}^{\prime \prime} * S_{\epsilon} * f_{\epsilon}^{\prime}\right)(t)-\left(r_{0}^{\prime \prime} * S_{0} * f^{\prime}\right)(t)\right\| \leq\left\|r_{0}^{\prime \prime}\right\|_{\infty}\left(A_{\epsilon}(t)+B_{\epsilon}(t)\right)
$$

Since

$$
A_{\epsilon}(t) \leq b M e^{\omega b} \int_{0}^{b}\left\|f_{\epsilon}^{\prime}(\tau)-f^{\prime}(\tau)\right\| d \tau
$$

by the stability condition (3.2). Then $\lim _{\epsilon \rightarrow 0^{+}} A_{\epsilon}(t)=0$ by (4.19). On the other side we have that

$$
B_{\epsilon}(t) \leq \int_{0}^{b}\left\|\left(\left(S_{\epsilon}-S_{0}\right) * f^{\prime}\right)(s)\right\| d s .
$$

Since $\left(\left(S_{\epsilon}-S_{0}\right) * f^{\prime}\right)(s) \rightarrow 0$ uniformly on $I_{b}$ by (4.20) implying that $B_{\epsilon}(t) \rightarrow 0$ as $\epsilon \rightarrow 0^{+}$. Thus $\left(r_{0}^{\prime \prime} * S_{\epsilon}^{\prime} * f_{\epsilon}\right)(t) \rightarrow\left(r_{0}^{\prime \prime} * S_{0}\right)(t)(f(0))+\left(r_{0}^{\prime \prime} * S_{0} * f^{\prime}\right)(t)$, and hence

$$
\lim _{\epsilon \rightarrow 0+}\left(r_{0}^{\prime} * S_{\epsilon}^{\prime \prime} * f_{\epsilon}\right)(t)=S_{0}(t)(f(0))+\left(S_{0} * f^{\prime}\right)(t)+\left(r_{0}^{\prime \prime} * S_{0}\right)(t)\left(f(0)+\left(r_{0}^{\prime \prime} * S_{0} * f^{\prime}\right)(t) .\right.
$$

Moreover, it is not hard to prove that

$$
S_{0}(t)(f(0))+\left(S_{0} * f^{\prime}\right)(t)+\left(r_{0}^{\prime \prime} * S_{0}\right)(t)\left(f(0)+\left(r_{0}^{\prime \prime} * S_{0} * f^{\prime}\right)(t)=\left(r_{0}^{\prime \prime} * S_{0}^{\prime} * f\right)(t)+\left(S_{0}^{\prime} * f\right)(t),\right.
$$

and this concludes with the proof of (4.18). Hence, we have shown that $\lim _{\epsilon \rightarrow 0^{+}} u_{\epsilon}(t)=u(t)$, where

$$
u(t):=w_{0}+\left(r_{0}^{\prime \prime} * S_{0}^{\prime} * f\right)(t)+\left(S_{0}^{\prime} * f\right)(t) .
$$

We remark that $u(t)$ can also be represented as

$$
u(t)=S_{0}^{\prime}(t) w_{0}+\left(r_{0}^{\prime \prime} * S_{0}\right)(t) w_{0}+\left(r_{0}^{\prime \prime} * S_{0}^{\prime} * f\right)(t)+\left(S_{0}^{\prime} * f\right)(t) .
$$

Since $A$ is closed then $A \omega_{0}=0$ by (H2). Thus $S_{0}^{\prime}(t) \omega_{0}=a_{0}(t) \omega_{0}$. On the other hand we recall that $\left(r_{0}^{\prime} * a_{0}\right)(t)=t$ by (4.5) then by differentiating on both sides follows that $\left(r_{0}^{\prime \prime} * a_{0}\right)(t)=1-a_{0}(t)$. Hence

$$
\begin{aligned}
\left(r_{0}^{\prime \prime} * S_{0}^{\prime}\right)(t) w_{0} & =\left(r_{0}^{\prime \prime} * a_{0}\right)(t) w_{0} \\
& =\left(1-a_{0}(t)\right) w_{0} .
\end{aligned}
$$

Therefore,

$$
\begin{aligned}
u(t) & =w_{0}+\left(S_{0} * f\right)(t)+\left(r_{0}^{\prime \prime} * S_{0} * f\right)(t) \\
& =a_{0}(t) w_{0}+\left(1-a_{0}(t)\right) w_{0}+\left(r_{0}^{\prime \prime} * S_{0}^{\prime} * f\right)(t)+\left(S_{0}^{\prime} * f\right)(t) \\
& =S_{0}^{\prime}(t) w_{0}+\left(r_{0}^{\prime \prime} * S_{0}^{\prime}\right)(t) w_{0}+\left(r_{0}^{\prime \prime} * S_{0}^{\prime} * f\right)(t)+\left(S_{0}^{\prime} * f\right)(t),
\end{aligned}
$$

proving the representation (4.23).

To finish the proof, we claim that $u(t)$ satisfy the integrated equation

$$
u(t)-w_{0}=A(1 * u)(t)+A(K * 1 * u)(t)+(1 * f)(t) .
$$


Once we have shown that $u(t)$ is a solution of the integrated equation we obtain that $u^{\prime}(t)$ exists and equation (1.2) is satisfied.

We notice, from identity (4.23) follows that

$$
(1 * u)(t)=S_{0}(t) w_{0}+\left(r_{0}^{\prime \prime} * S_{0}\right)(t) w_{0}+\left(r_{0}^{\prime \prime} * S_{0} * f\right)(t)+\left(S_{0} * f\right)(t)
$$

and since $r_{0}^{\prime \prime}(t)+K(t)+\left(r_{0}^{\prime \prime} * K\right)(t)=0$, then the right hand side of the integrated equation (4.24) equals to

$$
\begin{aligned}
J(t) & :=A(1 * u)(t)+K * A(1 * u)(t)+(1 * f)(t) \\
& =\left(r_{0}^{\prime \prime} * A S_{0} * f\right)(t)+\left(A S_{0} * f\right)(t)+\left(r_{0}^{\prime \prime} * K * A S_{0} * f\right)(t)+\left(K * A S_{0} * f\right)(t)+(1 * f)(t) \\
& \left.=\left(r_{0}^{\prime \prime} * A S_{0} * f\right)(t)+\left(A S_{0} * f\right)(t)+\left(\left(r_{0}^{\prime \prime} * K\right)+K\right) * A S_{0} * f\right)(t)+(1 * f)(t) \\
& =\left(r_{0}^{\prime \prime} * A S_{0} * f\right)(t)+\left(A S_{0} * f\right)(t)-\left(r_{0}^{\prime \prime} * A S_{0} * f\right)(t)+(1 * f)(t) \\
& =\left(A S_{0} * f\right)(t)+(1 * f)(t)
\end{aligned}
$$

Since $\left(r_{0}^{\prime \prime} * a\right)(t)=1-a_{0}(t)$ and hence we have that $\left(r_{0}^{\prime \prime} * S_{0}^{\prime} * f\right)(t)+\left(S_{0}^{\prime} * f\right)(t)=\left(A S_{0} * f\right)(t)+(1 * f)(t)$. Then we can write $(4.22)$ as $u(t)-w_{0}=A S_{0}(t)+\left(A S_{0} * f\right)(t)+(1 * f)(t)$; hence we have shown that $u(t)-w_{0}=J(t)$, and the proof is now finished.

\section{REFERENCES}

[1] R. Chill, A. Haraux. An optimal estimate for the difference of solutions of two abstract evolution equations. J. Differ. Equations. 193 (2) (2003), 385-395.

[2] K.-J. Engel. On singular perturbations on second order Cauchy problems . Pacific J. Math. 152 (1) (1992), 79-91.

[3] V. Kéyantuo. The Laplace transform and the ascent method for abstract wave equations J. Diff. Equations, 122 (1) ( 1995), 27-47.

[4] J. Liang, J. Liu, and T. Xiao. Hyperbolic singular perturbation for integrodifferential equations. Appl. Math. Comp. 163 (2005) 609-620.

[5] J.H. Liu. Singular perturbations of integrodifferential equations in Banach space Proc. Amer. Math. Soc. 122 (3) (1994), 791-799.

[6] C. Lizama. Regularized solutions for abstract Volterra equations. J. Math. Anal. Appl. 243 (2000), 278-292.

[7] C. Lizama. On approximation and representation of k-regularized resolvent families. Integr. Equ. Oper. Theory 41 (2001) 223-229.

[8] C.Lizama, H. Prado. Singular perturbation of integro-differential equations. Appl. Math. Comp. to appear.

[9] J. Prüss.Positivity and regularity of hyperbolic Volterra equations in Banach spaces. Math. Ann. 279 (1987), 317-344.

[10] J. Prüss. Evolutionary Integral Equations and Applications. Monographs Math., 87, Birkhäuser Verlag, 1993.

[11] M. Renardy, W. J. Hrusa, J.A. Nohel.Mathematical Problems in Viscoelasticity. Pitman Monographs Pure Appl. 35,Math. Longman Sci. Tech., Harlow, Essex, 1988.

Universidad de Santiago de Chile, Departamento de Matemática, Casilla 307-Correo 2, Santiago, CHILE

E-mail address: clizama@usach.cl

Universidad de Santiago de Chile, Departamento de Matemática, Casilla 307- Correo 2, Santiago, CHILE

E-mail address: hprado@usach.cl 